\title{
Comportamientos de riesgo y abuso sexual en varones y mujeres con discapacidad visual o auditiva
}

\author{
Risk behaviors and sexual abuse among \\ men and woman with visual or hearing \\ impairments
}

\section{Resumen}

El propósito del estudio fue analizar las características sociodemográficas, familiares y de comportamientos de riesgo y de abuso sexual de una muestra de jóvenes mexicanos con discapacidad auditiva o visual, examinando las diferencias por sexo y tipo de discapacidad. Se trabajó con I 28 jóvenes (64 con discapacidad auditiva y 64 con discapacidad visual) de los que un 53,2\% eran varones y un $86,6 \%$ solteros. Los instrumentos fueron adaptados lingüísticamente para ser respondidos por jóvenes con discapacidad auditiva y traducidos al braille para los jóvenes con discapacidad visual. Los resultados muestran que los participantes mantienen una vida sexual activa con un perfil de riesgo que se distingue por el inicio temprano de las relaciones sexuales, la falta de planificación de su primer encuentro sexual, no usar el preservativo y haber sufrido algún tipo de abuso sexual. El riesgo fue mayor en las mujeres y en quienes presentan discapacidad visual.

\section{Palabras clave}

Abuso sexual, discapacidad, jóvenes, conductas sexuales de riesgo.

\begin{abstract}
The present study proposed a descriptive analysis of sociodemographic and family variables, as well as risky sexual behaviors and sexual abuse in a sample of young Mexicans with hearing or visual impairments, examining differences based on gender and type of disability of the participants. I 28 young persons $(64$ hearing impaired and 64 visually impaired), of whom $53.2 \%$ were male and $86.6 \%$ were single, were included in the study. The instruments used were adapted linguistically to be answered by young people with hearing disabilities, and translated into Braille for visually impaired young people. The results show that participants maintain an active sex life with a risk profile that is characterized by the early onset of sexual intercourse, lack of planning their first sexual encounter, underuse of condoms and victimization of some form of sexual abuse. The risk was higher in women and among those with visual disabilities.
\end{abstract}

\section{Keywords}

Sexual abuse, impairment, youth, risky sexual behavior.

\section{Silvia Susana Robles Montijo \\ <susana@unam.mx>}

Universidad Nacional Autónoma de México

\section{Carmen Yolanda Guevara \\ Benítez}

<yolaguevara@hotmail.com>

Universidad Nacional Autónoma de México

Para citar:

Robles, S. S. y Guevara, C. Y. (20I 7): "Comportamientos de riesgo y abuso sexual en varones y mujeres con discapacidad visual o auditiva". Revista Española de Discapacidad, 5 (I): I33-I 5 I.

Doi: <https://doi.org/IO. 5569/23405 I04.05.01.08>

Fecha de recepción: 24-09-20I6

Fecha de aceptación: I7-04-20I7 


\section{Introducción ${ }^{1}$}

Las investigaciones sobre la sexualidad de adolescentes y jóvenes han permitido desarrollar una caracterización de conductas y factores afectivos. Autores como López Sánchez et al. (20II) exponen que los estudios realizados en diversos países demuestran que existe un doble patrón sexual y afectivo que inicia en la niñez y se mantiene durante la adolescencia y juventud. La sexualidad de las niñas y jóvenes mujeres es más afectivo-relacional, mientras que la sexualidad de los varones está más relacionada con la excitación y el placer sexual. La conducta de masturbación la practica un número mucho mayor de varones que de chicas, haciéndolo además con mayor frecuencia; ellos también suelen estar más dispuestos al sexo ocasional, sin afectos o compromisos. Ellas encuentran mayor placer en la comunicación, las caricias y los juegos afectivo-sexuales, por lo que suelen tener menos parejas, mientras que ellos buscan directamente la conducta coital. Las chicas suelen quejarse del machismo de los chicos, su falta de delicadeza y de que busquen el coito directamente, mientras ellos suelen quejarse de la resistencia de las chicas para aceptar tener relaciones coitales.

Los estudios que analizan relaciones de noviazgo han ubicado la presencia de patrones de abuso psicológico, físico y sexual en adolescentes (entre I 6 y 26 años de edad). Muñoz-Rivas et al. (2009) exponen que las estimaciones de victimización sexual hacia las jóvenes por parte de su novio se ubican en un rango entre el I $4 \%$ y el $43 \%$, mientras que el caso contrario puede ubicarse en un rango entre el o,3\% y

I. Este artículo se deriva de una investigación denominada "Factores de riesgo para la salud sexual de jóvenes con discapacidad visual y auditiva: un estudio exploratorio" financiada por el Programa de Apoyo a Proyectos de Investigación e Innovación Tecnológica (PAPIIT-DGAPA-UNAM), clave $\mathrm{IN}_{3} \mathrm{O}_{5} \mathrm{I}_{3}$, realizado desde enero de $20 \mathrm{I}_{3}$ hasta diciembre de 2014 .

Agradecimientos: las autoras agradecen a Irma Beatriz Frías Arroyo, Martha Rodríguez Cervantes, Odhet Daniela Medina Vargas y Nuvia Alejandra Cisneros Hernández por su valiosa colaboración en la aplicación de los instrumentos de evaluación. el $36 \%$. El estudio realizado en España por estos autores corroboró que el porcentaje de víctimas de agresión sexual es muy superior para las mujeres; sus resultados muestran que tanto la agresión como la victimización sexual son fundamentalmente de naturaleza psicológica, incluyendo la utilización de tácticas coercitivas de naturaleza verbal, como insistir en la relación coital sin que ella lo deseara. Los datos mencionados son consistentes con los de múltiples investigaciones sobre abuso: los victimarios son, en su gran mayoría, hombres. "La victimización sexual puede ser tan común en nuestra sociedad debido al grado de supremacía masculina que existe. Es una manera en que los hombres, el grupo de calidad dominante, ejercen control sobre la mujer" (Finkelhor, 2008: 47).

Según la Organización Mundial de la Salud (OMS, 20I6a), los adolescentes y jóvenes presentan un cuadro de múltiples riesgos. Se calcula que en 2015 murieron I, 3 millones de adolescentes, en su mayoría por causas prevenibles o tratables. Las causas más comunes de mortalidad son el virus de inmunodeficiencia humana (VIH), el suicidio y la violencia interpersonal. Entre los principales problemas de salud se ubican los embarazos y partos precoces, los abortos y las infecciones de transmisión sexual (ITS). Muchos de los problemas de salud en adolescentes y jóvenes pueden prevenirse con asesoría médica y psicológica sobre el tipo de relaciones amorosas y sobre el uso correcto y consistente del preservativo.

En México, los datos disponibles coinciden con los reportados por la OMS para la población mundial de adolescentes y jóvenes. La Encuesta Nacional de Salud y Nutrición (Gutiérrez et al., 2013) indicó que el $25 \%$ de los chicos y el $20 \%$ de las chicas adolescentes han iniciado su vida sexual; el $20 \%$ de ellos y el $39 \%$ de ellas reportaron no haber utilizado preservativo en su primera relación coital, y una mayor proporción (22 \% y $54 \%$, respectivamente) no lo utilizaron en su última relación. Es importante aclarar que el $90 \%$ de los adolescentes encuestados dijo conocer o haber escuchado sobre métodos anticonceptivos. 
Algunos factores que ponen en riesgo la salud se relacionan con las circunstancias particulares de la primera relación coital, como la edad en que se presentó, el tipo de pareja, el lugar y su planificación. Estudios realizados con adolescentes y jóvenes mexicanos indican que, en promedio, ellos inician su vida sexual entre los I 4 y los I7 años de edad, la pareja más frecuente es el novio/a y el lugar suele ser la casa del novio/a. Cerca de la mitad de los participantes no planificaron ese primer encuentro coital ni usaron algún método anticonceptivo (Andrade et al., 2006; DíazLoving y Robles, 2009; Enríquez et al., 2005; Robles et al., 2006, 20I I); cuando los jóvenes no planifican la primera relación sexual suelen no usar preservativo (Moreno et al., 2008).

Otros datos estadísticos en México son proporcionados por el Centro Nacional para la Prevención y Control del VIH y el SIDA (CENSIDA, 20I 5 ). Entre los casos de personas que viven con VIH se detectaron 3 I 77 jóvenes de I 5 a I9 años de edad ( $5,5 \%)$, II.324 entre 20 y 24 años (I9,7\%) y I 2699 (2I,4\%) entre 25 y 29 años. Se estima que este padecimiento provoca una tasa de mortandad de 4,2 por cada I00.000 habitantes. Por su parte, el Instituto Nacional de Estadística, Geografía e Informática (INEGI, 20I5) reporta un incremento sostenido en los últimos cinco años, en los nacimientos de niños cuyas madres son menores a 20 años, llegando al I9 \% de los nacimientos en el país. Mientras que la Secretaría de Salud de la Ciudad de México (2017) registra un total de $\mathrm{I} 77.604$ casos acumulados de abortos legales desde abril de 2007 hasta mayo de 2017. La mayor proporción de dichos abortos correspondió a mujeres entre I 8 y 24 años $(47, \mathrm{I} \%$ ) y el $5,6 \%$ a adolescentes entre I I y I 7 años de edad.

Desafortunadamente, en ningún caso se conoce qué proporción de dichas cifras corresponde a personas con discapacidad ni cuál es el perfil de las prácticas sexuales de riesgo en esa población. Posiblemente, esto se deba a la poca importancia que otorga la sociedad a la salud reproductiva de las personas con discapacidad, dada la creencia todavía extendida en amplias capas de la sociedad de que no son sexualmente activas y no necesitan cuidar su salud sexual. Sin embargo, se ha documentado que los jóvenes con discapacidad visual y auditiva pueden mantener una vida sexual activa y que, por carecer de educación sexual, su nivel de exposición a los factores de riesgo asociados con ITS, VIH y embarazos no deseados puede ser superior al de la población en general (Berman et al., 20I I; Sargowawa et al., 2009). Según un estudio realizado por la organización Save the Children (2003), en 205 instituciones latinoamericanas de salud se admitió haber atendido numerosos casos de abuso sexual en agravio de niños y jóvenes con discapacidad, muchos de los cuales tuvieron como resultado ITS y embarazos, pero reconocieron que tales datos no se registran ni se sistematizan. Ante esas circunstancias, la población con discapacidad es considerada en grave riesgo de embarazo, ITS y abuso sexual (Ferreira, 2008; Moinester et al., 2008).

El abuso sexual se define como "cualquier solicitud o ejercicio de contacto, caricias, juegos o toqueteos, en los que al menos uno de los implicados no desea, conoce o carece de conciencia de lo que está pasando, y que se obtiene por la fuerza o la ascendencia sobre la víctima" (Rodríguez et al., 20I 2: 59). El abuso sexual infantil (ASI) incluye todo el espectro de crímenes y delitos sexuales en los que son víctimas los niños hasta la edad de diecisiete años. "Abarca ciertos tipos de delitos sin contacto, como el exhibicionismo y el uso de niños en la producción de pornografía, así como delitos relacionados con la delincuencia sexual, además de los actos sexuales y de penetración que constituyen la mayoría de los casos" (Finkelhor, 2009: 7I).

Aunque la prevalencia del abuso sexual no se conoce con certeza, la OMS (20I6b) reporta que una de cada cinco mujeres y uno de cada trece hombres declaran haber sufrido abusos sexuales en la infancia. Algunos estudios permiten ubicar en la población general de diversos países tasas de entre el $7 \%$ y el $36 \%$ (Cantón y Cortés, 2015; Ocampos, 2005). En América Latina, uno de cada cinco niños son víctimas 
de abuso sexual (Rodríguez et al., 20I2). Las investigaciones de diversos autores permitieron ubicar que quienes llevan a cabo el abuso, con mayor frecuencia, son amigos, vecinos o parientes de la víctima que, en su mayoría, no se comportan con brutalidad o sadismo, sino que usan su autoridad o su encanto para ganarse la confianza, cooperación y pasividad del menor. Sus prácticas más comunes consisten en tocar los genitales, el exhibicionismo, la masturbación y la penetración, aunque también se pueden presentar violaciones que pueden ser brutales (Finkelhor, 2008). Las consecuencias del abuso sexual en las víctimas pueden ser físicas y psicológicas, incluyendo ITS, problemas emocionales y conducta sexualizada. Entre los indicadores de que un menor ha sufrido abuso se pueden ubicar: conducta sexual precoz, hablar constantemente sobre sexo, agresividad sexual, conducta seductora, lenguaje obsceno, masturbación frecuente, interacción sexual con objetos, exhibicionismo, intentos de juego sexual frecuente con sus pares u otras personas (González et al., 2012).

El abuso sexual ha sido poco estudiado en personas con discapacidad. La investigación realizada por Smith (2008) comparó situaciones de abuso en poblaciones estadounidenses de hombres y mujeres con discapacidad y sin ella. Los resultados indicaron que hombres y mujeres con discapacidad viven diversas formas de violencia -incluyendo amenazas, intentos, violencia física real, así como relaciones sexuales no deseadas-, pero que son las mujeres con discapacidad quienes viven estas situaciones con mayor frecuencia. Nosek et al. (200I) también probaron que estas mujeres experimentan altos niveles de abuso emocional, físico y sexual. Entre las razones por las que ellas viven una mayor vulnerabilidad al abuso destacan: un mayor grado de dependencia para su cuidado; la negación de sus derechos humanos, asociada con la desvalorización cultural hacia las mujeres y hacia las personas con discapacidad; su propia percepción de impotencia y baja auto-estima; su mínima o nula educación sobre la sexualidad apropiada e inapropiada; aislamiento social y mayor riesgo de manipulación; desamparo físico y vulnerabilidad en lugares públicos.
Muchos de esos riesgos están estrechamente relacionados con el hecho de vivir en situaciones de sobreprotección familiar y de negación de su sexualidad, además de la existencia de estereotipos y expectativas sociales reducidas.

El abuso sexual perpetrado con personas que viven situaciones de discapacidad es un tema poco documentado en sociedades latinoamericanas. Santín (2OI2) expone que muchos casos atendidos en los centros de rehabilitación han presentado alteraciones en la sexualidad y en su comportamiento, en los que parecen reproducir escenas de abuso sexual, excesiva manipulación, desinhibición o agresión, entre otros rasgos que no son esperados para su nivel de desarrollo. Según esta autora, la prevalencia del abuso sexual hacia estas personas es mayor a la de la población sin discapacidad, pero muchas veces no se presentan denuncias, entre otras razones por las limitaciones comunicativas de los afectados.

Sakellariou (2006) explica que las personas con discapacidad son vistas por la sociedad bajo un prisma paternalista, como necesitadas de protección y sin impulsos sexuales. Como resultado, su sexualidad es un tema tabú que enfrenta una fuerte resistencia social. Dicho panorama se evidencia en muchas familias de personas con discapacidad. Guevara et al. (20I6a) señalan que es a través de la familia que se logra un proceso de integración social del individuo, incluyendo: aprendizaje de conceptos y de reglas sociales, formas de interactuar con el ambiente físico y social, y desarrollo de patrones emocionales y de conducta. Para ello, cada familia adopta estilos de crianza particulares. Aquellos niños criados en un sistema autoritario, que suele caracterizar a las familias de niños y jóvenes con discapacidad, con frecuencia pueden estar expuestos a peligros, porque están acostumbrados a obedecer, no a tomar decisiones.

Entre las situaciones que ponen en riesgo a esta población están: las relaciones de dependencia y sumisión que suelen caracterizar las interacciones que establecen con sus padres y cuidadores, la pérdida de intimidad que 
facilita el abuso, la ausencia de un modelo de rol sexual, la falta de información sobre salud sexual, la imposibilidad de distinguir entre la adecuación o no de ciertas conductas relacionadas con la sexualidad, la falta de habilidades socio-sexuales y contar con amistades que no tienen discapacidad (Verdugo et al., 2002). Las personas con discapacidad se enfrentan a una serie de barreras que los llevan a limitaciones físicas, comunicativas, educativas y de integración social y familiar, incluyendo una educación sexual inadecuada o incluso ausente. Este panorama conduce a la perpetuación de mitos y a prácticas sexuales peligrosas, embarazos no deseados, miedos y expectativas poco realistas (Sakellariou, 2006). No debe dejarse de lado que, a pesar del aislamiento en que pueden vivir, estas personas reciben información sobre el papel social de una mujer y un hombre, junto con los valores que la sociedad les atribuye, lo que puede llevarlos a desear "un estándar de vida adulta" que incluye el ejercicio de su sexualidad e incluso de la maternidad, situación que conduce a un conflicto porque esos ideales y estándares no corresponden con la percepción que tienen de su propia persona y sus condiciones de vida (Parchomiuk, 20I4).

Según Campero et al. (20I3), las principales vías por las cuales los adolescentes sin discapacidad suelen recibir información sobre sexualidad son, en ese orden: medios de comunicación, amigos, profesores, literatura impresa y comunicación con padres. Todas esas vías de información suelen estar limitadas para los jóvenes con discapacidad visual y auditiva, porque los materiales que pueden instruirlos en estos temas no son accesibles para ellos, al menos en países como México, en donde los materiales en braille o los materiales a los que pueden acceder en Lengua de Señas Mexicana (LSM) para sordos, no están dirigidos a brindarles información sobre educación sexual. Debe considerarse, además, que sus padres no necesariamente desean abordar dichos temas o no saben cómo hacerlo (García y Nastri, 20I I; Robles et al., 20I3), y que sus profesores no cuentan con la autorización de los padres o no cubren dichos temas porque no forman parte del plan curricular.
También debe tomarse en cuenta que la población de jóvenes mexicanos con discapacidad visual y auditiva cuenta con un promedio de escolaridad de 3,5 grados, sólo el $48 \%$ asiste a la escuela y el $32 \%$ es analfabeta (INEGI, 20I 5). La falta de educación en general y de educación sexual en particular lleva a estos jóvenes a una situación de desconocimiento de muchos aspectos básicos, incluyendo la forma de tratar con extraños, cómo ubicar una situación de abuso, cómo negarse a esta situación o cómo reportarla. Asimismo, pueden carecer de habilidades para tomar decisiones sociales/sexuales, vivir condiciones que no pueden controlar y ni siquiera plantearse que las prácticas sexuales desprotegidas pueden ponerlos en situaciones de riesgo de ITS, VIH/ SIDA y embarazos no deseados (Kochung y Were, 20I I; Pontes y Freitag, 20I0).

En México no se cuenta con estadísticas que den cuenta de los problemas de salud sexual de jóvenes con discapacidad auditiva o visual. Probablemente, una de las razones de esta ausencia sea la complejidad implicada en el diseño de instrumentos dirigidos a esta población ya que, en el caso de las personas sordas, se requiere diseñar instrumentos adaptados a las características lingüísticas de esta población, mientras que en el caso de las personas con discapacidad visual se necesita traducir los instrumentos al sistema braille.

Entre los escasos estudios sobre el tema se encuentra el reportado por Robles et al. (20I4), quienes construyeron y adaptaron una serie de instrumentos para ser respondidos por jóvenes con discapacidad auditiva, así como el estudio de Guevara et al. (20I6b) en el que se reporta su adaptación para ser aplicados a jóvenes con discapacidad visual. Tales instrumentos permiten evaluar: datos sociodemográficos y familiares, antecedentes de la discapacidad, comunicación con padres y con madres sobre temas relacionados con el cuidado de la salud sexual, apoyo y supervisión parental, conocimientos sobre VIH, ITS y embarazo, conocimientos sobre el uso correcto del preservativo, aspectos relacionados con su primera experiencia coital (edad, planeación, lugar, tipo de pareja y uso de 
algún método anticonceptivo) y con experiencias coitales subsecuentes (frecuencia de relaciones con penetración vaginal, frecuencia de uso del preservativo, fallas en su uso, relaciones con parejas ocasionales, uso del preservativo en dichas relaciones y número de parejas sexuales en toda la vida), incluyendo prácticas de abuso sexual sufrido por los jóvenes y reporte de VIH, ITS, embarazo y aborto (Robles et al., 201 5 ).

Con esos antecedentes, el presente estudio se propuso realizar un análisis descriptivo de variables sociodemográficas, familiares y de prácticas de riesgo y de abuso sexual en una muestra de jóvenes con discapacidad auditiva o visual, examinando las diferencias en función del sexo y tipo de discapacidad de los participantes. Consideramos que este propósito puede contribuir con indicadores que den cuenta de las posibles diferencias entre varones y mujeres y entre quienes tienen discapacidad visual y discapacidad auditiva, sugiriendo estrategias de prevención dirigidas especialmente a cada grupo de jóvenes con discapacidad.

\section{Método}

De acuerdo con Méndez et al. (200I), el estudio fue de tipo transversal-descriptivo-comparativoobservacional, ya que se llevó a cabo la aplicación de los instrumentos en una sola ocasión, se realizaron análisis descriptivos de las variables y se exploró su asociación con el sexo y el tipo de discapacidad de los participantes sin llevar a cabo manipulaciones experimentales.

\section{- Participantes}

Los instrumentos se aplicaron a I74 jóvenes con discapacidad auditiva (DA) y 64 con discapacidad visual (DV), elegidos a través de un muestreo accidental realizado en Io instituciones educativas y cuatro centros deportivos de la zona metropolitana de la Ciudad de México que atienden a personas con discapacidad. Dado que uno de los propósitos de este estudio fue analizar las diferencias en el comportamiento sexual de los jóvenes en función del sexo y

\begin{tabular}{|c|c|c|c|c|c|c|c|c|c|}
\hline \multirow{3}{*}{ Característica } & \multirow{2}{*}{\multicolumn{2}{|c|}{$\begin{array}{c}\begin{array}{c}\text { Toda la } \\
\text { muestra }\end{array} \\
(\mathrm{N}=128)\end{array}$}} & \multicolumn{4}{|c|}{ Discapacidad } & \multirow[b]{3}{*}{$\mathbf{X}^{2}$} & \multirow[b]{3}{*}{ gl } & \multirow[b]{3}{*}{$p$} \\
\hline & & & \multicolumn{2}{|c|}{$\begin{array}{l}\text { auditiva } \\
(n=64)\end{array}$} & \multicolumn{2}{|c|}{$\begin{array}{l}\text { visual } \\
\text { (n=64) }\end{array}$} & & & \\
\hline & $\mathbf{n}$ & $\%$ & $\mathbf{n}$ & $\%$ & $\mathbf{n}$ & $\%$ & & & \\
\hline \multicolumn{10}{|l|}{ Sexo } \\
\hline Hombre & 64 & 50,0 & 32 & 50,0 & 32 & 50,0 & .000 & 2 & 1.00 \\
\hline Mujer & 60 & 46,9 & 30 & 46,9 & 30 & 46,9 & & & \\
\hline Sin respuesta & 4 & 3.1 & 2 & 3.1 & 2 & 3.1 & & & \\
\hline \multicolumn{10}{|l|}{ Edad } \\
\hline Entre 12 y 14 & 4 & 3,1 & 1 & 1,6 & 3 & 4,7 & 6.576 & 6 & .362 \\
\hline Entre 15 y 17 & 20 & 15,6 & 11 & 17,2 & 9 & 14,1 & & & \\
\hline Entre 18 y 20 & 28 & 21,9 & 15 & 23,4 & 13 & 20,3 & & & \\
\hline Entre 21 y 23 & 37 & 28,9 & 22 & 34,4 & 15 & 23,4 & & & \\
\hline Entre 24 y 26 & 16 & 12,5 & 8 & 12,5 & 8 & 12,5 & & & \\
\hline Más de 26 años & 22 & 17,2 & 7 & 10,9 & 15 & 23,4 & & & \\
\hline Sin respuesta & 1 & .8 & 0 & 0 & 1 & 1,6 & & & \\
\hline
\end{tabular}

Fuente: elaboración propia. 
tipo de discapacidad, se consideró conveniente conformar dos grupos homogéneos tomando en cuenta ambas características. Por ello, de los I74 jóvenes con DA se seleccionó una muestra de 64 que fuera semejante al grupo con DV en el número de hombres y mujeres y en la edad de los participantes (Tabla I). De esta manera, la muestra final estuvo conformada por I 28 jóvenes (64 con DA y 64 con DV); la mayoría eran solteros $(86,6 \%)$ y vivían con uno o ambos padres $(7 \mathrm{I}, 6 \%)$; el 30,4 \% eran estudiantes de secundaria, el $45,6 \%$ de preparatoria y el Io,4 \% de educación superior, mientras que el I, $6 \%$ se dedicaba a otras actividades u oficios, y el $4 \%$ carecía de estudios; el 34,2 \% trabajaba en el momento en que se aplicaron los instrumentos.

\section{- Instrumentos}

Los instrumentos que evalúan datos sociodemográficos, datos familiares y abuso sexual fueron construidos y adaptados lingüísticamente para ser respondidos en papel por jóvenes con discapacidad auditiva o visual. El proceso de adaptación y su validación a la población objetivo se reporta en los trabajos de Robles et al. (2013) para jóvenes con DA, y Guevara et al. (20I6b) para jóvenes con DV. El instrumento que evalúa prácticas de riesgo se retomó del trabajo de Robles y Díaz-Loving (2OII) validado con población oyente, y también fue adaptado por Robles et al. (20I3) y Guevara et al. (20I6b) para ser respondido por jóvenes con DA y DV, respectivamente.

- Datos sociodemográficos. El instrumento consta de seis reactivos para obtener información de los participantes acerca de su edad (entre I 2 y I4, I 5 y I7, I 8 y 20, 2 I y 23, 24 y 26, y más de 26 años), sexo (hombre-mujer), estado civil (soltero, casado, unión libre y otros), con quién viven (casa paterna/materna, de otro familiar, internado o propia casa), nivel de escolaridad (desde primaria hasta universidad, incluyendo oficios), y si trabaja o no.

- Datos familiares. El instrumento está conformado por once reactivos que permiten obtener información acerca de los progenitores de los participantes: si ambos viven (sí-no), si viven juntos o separados (si-no), su edad (menos de 30 años, entre 30 y 40 , entre 4 I y 50 , entre 5 I y 60 , más de 60), su nivel de escolaridad (sin estudios, con algún oficio, primaria, secundaria, preparatoria y universidad), su situación laboral (si trabajan o no) y si presentan alguna discapacidad (visual, auditiva, motriz, otra, sin discapacidad).

- Prácticas de riesgo. Se utilizaron once reactivos para obtener información de los jóvenes acerca de si han tenido o no relaciones coitales, características de su primera relación coital considerando edad (menos de I4 años, entre I 5 y I7, entre I8 y 20 , entre 2 I y 23 y entre 24 y 26 ), si fue planificada (si-no), el lugar en la que ocurrió (casa del participante, del novio/a, de un amigo/a, de un familiar o en un hotel), tipo de pareja (novio/a, amigo/a, un familiar o alguna persona desconocida), uso de algún método anticonceptivo (si-no), tipo de método anticonceptivo (preservativo, píldoras anticonceptivas, no eyacular cuando se tiene la relación coital, tener sexo anal, otro), número de parejas con quienes han tenido alguna relación coital (ninguna, una, dos, tres, cuatro o más), número de relaciones coitales en toda la vida (una, entre 2 y 4 , entre 5 y 7 , entre 8 y IO, entre I I y I 5 , entre I 6 y 20 , más de 20), el uso del preservativo en dichas relaciones (con las mismas opciones de respuesta), tener relaciones con parejas ocasionales (sólo una, entre 2 y 4 , entre 5 y 7 , entre 8 y Io y más de Io) y con personas del mismo sexo (sí-no). El instrumento incluye también cuatro reactivos para preguntar a los jóvenes si han tenido algún embarazo, algún aborto, alguna ITS y si se han realizado pruebas para identificar éstas, con opciones de respuesta si-no.

- Abuso sexual. Este instrumento consta de cinco reactivos que, de acuerdo con Rodríguez et al. (2OI2), dan cuenta de situaciones de abuso sexual sufrido por los participantes, dos de ellos hacen referencia a si los obligaron a tener relaciones sexuales 
en su primera o en posteriores relaciones, y tres reactivos hacen referencia a obligarlos a tocar sus genitales o los de otra persona, o a quitarse la ropa, todos con opciones de respuesta dicotómicas (sí-no).

\section{- Procedimiento}

A las autoridades de las instituciones participantes se les informó acerca de los propósitos de la investigación. Una vez obtenida su anuencia para aplicar los instrumentos, se acordaron las fechas y horarios para acudir a realizar la evaluación. Cuatro instructores previamente capacitados en el manejo y aplicación de los instrumentos explicaron brevemente el propósito del estudio a los jóvenes y a sus padres, enfatizando la necesidad de su participación voluntaria y asegurándoles el tratamiento confidencial y anónimo de la información recabada. A los adultos que aceptaron participar se les solicitó la firma del consentimiento informado y, en el caso de los menores de edad, además de su aceptación se recabó la firma de autorización de uno de los padres. Los instrumentos se aplicaron grupalmente y fueron respondidos en forma individual. Los instructores explicaron a los participantes los contenidos y la forma de responder a ellos. En población sorda se contó con el apoyo de un intérprete de la Lengua de Señas Mexicana. Los jóvenes tardaron en responder entre 30 y 40 minutos.

\section{- Condiciones éticas del estudio}

El presente estudio fue revisado y avalado por el Comité de Bioética de la Facultad de Estudios Superiores Iztacala de la Universidad Nacional Autónoma de México y contó con el consentimiento informado de los padres de familia y de los propios jóvenes con discapacidad auditiva o visual. En las instrucciones generales para responder a los instrumentos se señaló expresamente que a los participantes no se les solicitaba su nombre y que si se sentían incómodos con las preguntas podían dejar de responder y entregar el cuestionario a los instructores.

\section{Resultados}

\section{- Datos familiares}

En la Tabla 2 se presentan los resultados correspondientes a los datos familiares de los jóvenes, en toda la muestra y por tipo de discapacidad. La información referida a los padres indicó que, en su mayoría, tenían entre 4I y 60 años de edad, su nivel máximo de estudios fue de secundaria y contaban con un trabajo remunerado. En el caso de las madres, las frecuencias más altas en cada variable indican una edad entre 4I y 50 años, un nivel de escolaridad de primaria y secundaria, y también contaban con un trabajo remunerado. La comparación de cada variable por tipo de discapacidad del participante mostró diferencias significativas sólo en la edad del padre $\left(\mathrm{X}^{2}=7.9 \mathrm{I} 4, \mathrm{gl}=3, \mathrm{p}=.04\right)$ y de la madre $\left(\mathrm{X}^{2}=\mathrm{I} 0.395, \mathrm{gl}=4, \mathrm{p}=.03\right)$, en el nivel de escolaridad de las madres $\left(\mathrm{X}^{2}=\mathrm{I} 4.076, \mathrm{gl}=5\right.$, $\mathrm{p}=. \mathrm{OI})$ y en la situación laboral del padre $\left(\mathrm{X}^{2}=\right.$ $4.050, \mathrm{gl}=\mathrm{I}, \mathrm{p}=.04)$. Cabe destacar que el tipo de discapacidad de ambos progenitores se asoció con el tipo de discapacidad de los jóvenes. Aun cuando la mayoría de los padres y de las madres no presentaba discapacidad, cuando ésta se ubicó correspondió con la presentada por el hijo(a) $\left(\mathrm{X}^{2}=\mathrm{I} 6.2 \mathrm{I} 2, \mathrm{gl}=3, \mathrm{p}=.00 \mathrm{I} \mathrm{y} \mathrm{X}^{2}=\right.$ $9.490, \mathrm{gl}=4, \mathrm{p}=.05$, respectivamente). 
Tabla 2. Datos familiares de toda la muestra y por tipo de discapacidad de los participantes

\begin{tabular}{|c|c|c|c|c|c|c|}
\hline \multirow[b]{2}{*}{ Característica } & \multicolumn{2}{|c|}{$\begin{array}{c}\text { Toda la muestra } \\
\qquad(\mathrm{N}=128)\end{array}$} & \multicolumn{2}{|c|}{$\begin{array}{l}\text { Discapacidad auditiva } \\
\qquad(\mathrm{N}=64)\end{array}$} & \multicolumn{2}{|c|}{$\begin{array}{c}\text { Discapacidad visual } \\
\qquad(\mathrm{N}=64)\end{array}$} \\
\hline & Frecuencia & $\%$ & Frecuencia & $\%$ & Frecuencia & $\%$ \\
\hline \multicolumn{7}{|l|}{ Vive el padre } \\
\hline Sí & 105 & 82.0 & 54 & 85.7 & 51 & 79.7 \\
\hline No & 22 & 17.2 & 9 & 14.3 & 13 & 20.3 \\
\hline \multicolumn{7}{|l|}{ Vive la madre } \\
\hline Sí & 120 & 93.8 & 59 & 96.7 & 61 & 95.3 \\
\hline No & 5 & 3.9 & 2 & 3.3 & 3 & 4.7 \\
\hline \multicolumn{7}{|c|}{ Ambos padres viven } \\
\hline Juntos & 73 & 75.3 & 41 & 82.0 & 32 & 68.1 \\
\hline Separados & 24 & 24.7 & 9 & 18.0 & 15 & 31.9 \\
\hline \multicolumn{7}{|l|}{ Edad del padre } \\
\hline Menos de 30 años & 0 & 0 & 0 & 0 & 0 & 0 \\
\hline Entre 30 y 40 & 10 & 9.9 & 2 & 4.0 & 8 & 15.7 \\
\hline Entre 41 y 50 & 46 & 45.5 & 29 & 58.0 & 17 & 33.3 \\
\hline Entre 51 y 60 & 32 & 31.7 & 14 & 28.0 & 18 & 35.3 \\
\hline Más de 60 & 13 & 12.9 & 5 & 10.0 & 8 & 15.7 \\
\hline \multicolumn{7}{|l|}{ Edad de la madre } \\
\hline Menos de 30 años & 2 & 1.7 & 2 & 3.6 & 0 & 0 \\
\hline Entre 30 y 40 & 18 & 15.7 & 11 & 20.0 & 7 & 11.7 \\
\hline Entre 41 y 50 & 57 & 49.6 & 30 & 54.5 & 27 & 45.0 \\
\hline Entre 51 y 60 & 21 & 18.2 & 9 & 16.4 & 12 & 20.0 \\
\hline Más de 60 & 17 & 14.8 & 3 & 5.5 & 14 & 23.3 \\
\hline \multicolumn{7}{|c|}{ Escolaridad del padre } \\
\hline Sin estudios & 8 & 7,8 & 2 & 3,9 & 6 & 11,8 \\
\hline Oficio & 4 & 3,9 & 0 & 0 & 4 & 7,8 \\
\hline Primaria & 23 & 22,5 & 9 & 17,6 & 14 & 27,5 \\
\hline Secundaria & 39 & 38,3 & 22 & 43,1 & 17 & 33,3 \\
\hline Preparatoria & 17 & 16,7 & 10 & 19,6 & 7 & 13,7 \\
\hline Universidad & 11 & 10,8 & 8 & 15,7 & 3 & 5,9 \\
\hline \multicolumn{7}{|c|}{ Escolaridad de la madre } \\
\hline Sin estudios & 17 & 14,5 & 8 & 14,3 & 9 & 14,8 \\
\hline Oficio & 5 & 4,3 & 0 & 0 & 5 & 8,2 \\
\hline Primaria & 33 & 28,2 & 10 & 17,9 & 23 & 37,7 \\
\hline Secundaria & 32 & 27,4 & 19 & 33,9 & 13 & 21,3 \\
\hline Preparatoria & 17 & 14,5 & 12 & 21,4 & 5 & 8,2 \\
\hline Universidad & 13 & 11,1 & 7 & 12,5 & 6 & 9,8 \\
\hline \multicolumn{7}{|l|}{ Trabaja el padre } \\
\hline Sí & 95 & 93,1 & 51 & 98,1 & 44 & 88,0 \\
\hline No & 7 & 6,9 & 1 & 1,9 & 6 & 12,0 \\
\hline \multicolumn{7}{|l|}{ Trabaja la madre } \\
\hline Sí & 78 & 66,7 & 38 & 66,7 & 40 & 66,7 \\
\hline
\end{tabular}




\begin{tabular}{|l|c|c|c|c|c|c|}
\hline & \multicolumn{2}{|c|}{$\begin{array}{c}\text { Toda la muestra } \\
\text { (N = 128) }\end{array}$} & \multicolumn{2}{c|}{$\begin{array}{c}\text { Discapacidad auditiva } \\
(\mathbf{N}=\mathbf{6 4})\end{array}$} & $\begin{array}{c}\text { Discapacidad visual } \\
\text { (N = 64) }\end{array}$ \\
\hline Característica & Frecuencia & $\%$ & Frecuencia & $\%$ & Frecuencia & $\%$ \\
\hline No & 39 & 33,3 & 19 & 33,3 & 20 & 33,3 \\
\hline Discapacidad del padre & & & & & & \\
\hline Sin discapacidad & 81 & 77,9 & 42 & 77,8 & 39 & 78,0 \\
\hline Visual & 13 & 12,5 & 2 & 3,7 & 11 & 22,0 \\
\hline Auditiva & 8 & 7,7 & 8 & 14,8 & 0 & 0 \\
\hline Otra & 2 & 1,9 & 2 & 3,7 & 0 & 0 \\
\hline Discapacidad de la madre & & & & & & \\
\hline Sin discapacidad & 102 & 83,5 & 49 & 80,3 & 53 & 86,9 \\
\hline Visual & 8 & 6,6 & 2 & 3,3 & 6 & 9,8 \\
\hline Auditiva & 7 & 5,7 & 7 & 11,5 & 0 & 0 \\
\hline Motriz & 2 & 1,6 & 1 & 1,6 & 1 & 1.6 \\
\hline Otra & 3 & 2,5 & 2 & 3,3 & 1 & 1,6 \\
\hline
\end{tabular}

Fuente: elaboración propia.

Se llevó a cabo una comparación por sexo para cada una de estas variables sociodemográficas y en ningún caso se encontraron diferencias significativas.

\section{- Comportamiento sexual}

Los resultados que se muestran en la Tabla 3 indican que el 61,7\% de la muestra había tenido relaciones coitales. La edad más frecuente de su primera relación coital fue entre los I 8 y los 20 años, sin embargo la suma de las frecuencias reportadas en los rangos "menos de I4" y “entre I 5 y I 7 años” indican que más de la mitad de los jóvenes $(52,7 \%)$ tuvo su primer coito antes de los i 8 años. La mayoría lo tuvo con su novio(a) o un amigo(a), pero cabe destacar que se registraron siete casos en los que la pareja en este primer coito fue un familiar o una persona desconocida. El lugar más frecuente del primer coito fue un hotel. Se encontró, además, que más de la mitad de los jóvenes sexualmente activos no planificaron su primer encuentro sexual y el 4I,9 \% no usó ningún método anticonceptivo. Entre quienes sí lo hicieron, el preservativo fue el método más utilizado.
Se estimó en toda la muestra el índice de correlación rho de Spearman entre planificar la primera relación coital y usar el preservativo en dicha relación, encontrándose una correlación positiva significativa $(r h o=0.3 \mathrm{I} 2, \mathrm{p}<.0 \mathrm{I})$. La comparación de cada variable por tipo de discapacidad mostró diferencias significativas únicamente en lo relativo al primer coito $\left(\mathrm{X}^{2}\right.$ $=4.00 \mathrm{I}, \mathrm{gl}=\mathrm{I}, \mathrm{p}=.04)$ y su planificación $\left(\mathrm{X}^{2}\right.$ $=\mathrm{I} 3 . \mathrm{I} 53, \mathrm{gl}=\mathrm{I}, \mathrm{p}<.00 \mathrm{I})$, con una mayor proporción de jóvenes con DV que ya habían tenido su primer coito $(70,3 \%)$ y que no lo planificaron $(69,8 \%)$, que los jóvenes con DA (53,I \% y 26,7\%, respectivamente). Sólo en el grupo de jóvenes con DV se encontró una correlación positiva significativa entre planificar el primer coito y usar el preservativo ( $r h o=$ $0.382, \mathrm{p}<.0 \mathrm{I})$, indicando que quienes sí lo planificaron también usaron condón, o bien que quienes no lo planificaron no lo utilizaron.

La comparación por sexo en las variables vinculadas con la primera relación coital mostró diferencias significativas $\left(\mathrm{X}^{2}=5 \cdot 100, \mathrm{gl}=\mathrm{I}, \mathrm{p}=\right.$ .02) en el uso de algún método anticonceptivo, con una proporción mayor en los hombres $(69,4 \%)$ que en las mujeres $(42,9 \%)$. También se encontraron diferencias significativas en el 
Tabla 3. Características de la primera relación coital en toda la muestra y por tipo de discapacidad de los participantes

\begin{tabular}{|c|c|c|c|c|c|c|}
\hline \multirow[b]{2}{*}{ Característica } & \multicolumn{2}{|c|}{$\begin{array}{c}\text { Toda la muestra } \\
\qquad(\mathrm{N}=128) \\
\end{array}$} & \multicolumn{2}{|c|}{$\begin{array}{l}\text { Discapacidad auditiva } \\
\qquad(64) \\
\end{array}$} & \multicolumn{2}{|c|}{$\begin{array}{c}\text { Discapacidad visual } \\
\text { (64) } \\
\end{array}$} \\
\hline & Frecuencia & $\%$ & Frecuencia & $\%$ & Frecuencia & $\%$ \\
\hline \multicolumn{7}{|l|}{ Experiencia coital } \\
\hline Sí & 79 & 61,7 & 34 & 53,1 & 45 & 70,3 \\
\hline No & 49 & 38,3 & 30 & 46,9 & 19 & 29,7 \\
\hline \multicolumn{7}{|l|}{ Edad del primer coito } \\
\hline Menos de 14 años & 10 & 13,5 & 4 & 13,3 & 6 & 13,6 \\
\hline Entre 15 y 17 años & 29 & 39,2 & 13 & 43,3 & 16 & 36,4 \\
\hline Entre 18 y 20 años & 31 & 42,9 & 13 & 43,3 & 18 & 40,9 \\
\hline Entre 21 y 23 años & 3 & 4,0 & 0 & 0 & 3 & 6,8 \\
\hline Entre 24 y 26 años & 1 & 1,3 & 0 & 0 & 1 & 2,3 \\
\hline \multicolumn{7}{|l|}{ Planificación } \\
\hline Sí & 35 & 47,9 & 22 & 73,3 & 13 & 30,2 \\
\hline No & 38 & 52,1 & 8 & 26,7 & 30 & 69,8 \\
\hline \multicolumn{7}{|l|}{ Lugar } \\
\hline Mi casa & 18 & 25,4 & 7 & 25,0 & 11 & 25,6 \\
\hline Casa de un amigo(a) & 12 & 16,9 & 2 & 7,1 & 10 & 23,3 \\
\hline Casa del novio(a) & 17 & 23,9 & 9 & 32,1 & 8 & 18,6 \\
\hline Hotel & 20 & 28,2 & 8 & 28,6 & 12 & 27,9 \\
\hline Casa de un familiar & 4 & 5,6 & 2 & 7,1 & 2 & 4,2 \\
\hline \multicolumn{7}{|l|}{ Tipo de pareja } \\
\hline Novio & 47 & 60,3 & 23 & 69,7 & 24 & 53,3 \\
\hline Amigo & 24 & 30,8 & 9 & 21,3 & 15 & 33,3 \\
\hline Familiar & 4 & 5,1 & 0 & 0 & 4 & 8,9 \\
\hline Desconocido & 3 & 3,8 & 1 & 3,0 & 2 & 4,4 \\
\hline \multicolumn{7}{|l|}{$\begin{array}{l}\text { Uso de algún método } \\
\text { anticonceptivo }\end{array}$} \\
\hline Sí & 43 & 58,1 & 19 & 61,3 & 24 & 55,8 \\
\hline No & 31 & 41,9 & 12 & 38,7 & 19 & 44,2 \\
\hline \multicolumn{7}{|c|}{ Método anticonceptivo usado } \\
\hline Ninguno & 29 & 39,8 & 12 & 38,7 & 17 & 40,5 \\
\hline Condón masculino & 36 & 49,3 & 16 & 51,6 & 20 & 47,6 \\
\hline Pastillas anticonceptivas & 6 & 8,2 & 2 & 6,5 & 4 & 9,5 \\
\hline No eyacular & 1 & 1,4 & 0 & 0 & 1 & 2,4 \\
\hline Sexo anal & 1 & 1,4 & 1 & 3,2 & 0 & 0 \\
\hline
\end{tabular}

Fuente: elaboración propia. 
tipo de método anticonceptivo utilizado $\left(\mathrm{X}^{2}=\right.$ I I.3 I I, $\mathrm{gl}=5, \mathrm{p}=.04)$, siendo el preservativo el método más utilizado por los hombres $(63,9 \%)$, comparados con las mujeres $(29,4 \%)$.

Respecto al comportamiento posterior a la primera relación coital, se encontró que el 45,6\% (n $=3 \mathrm{I})$ sólo había tenido una pareja sexual, el 20,6\% (n = I4) dos parejas y el 33,8\% (n $=23$ ) tres o más parejas. En relación con la frecuencia de las relaciones coitales en toda la vida, se encontró que el 35,6\% tuvo entre 2 y 7 y el $22 \%$ más de 20 relaciones coitales. El uso del preservativo en dichas relaciones fue menos frecuente, ya que el I 2,9\% no lo usó en ninguna de sus relaciones, el I 6,I \% lo usó sólo una vez, el 25,8\% entre 2 y 4 veces, el 9,7\% entre 5 y 7 veces, el $6,5 \%$ entre 8 y io veces, el $6,5 \%$ entre I I y I 5 veces y el $22,6 \%$ más de I 6 veces. El I7,9\% de los participantes tuvo relaciones coitales con personas de su mismo sexo, el 50,8\% tuvo relaciones coitales con parejas ocasionales o casuales y el 59,7\% nunca usó el preservativo en dichas relaciones. Sólo en esta última variable se encontraron diferencias significativas por tipo de discapacidad, ya que el 73,7\% de los jóvenes con DA reportó nunca haber tenido relaciones coitales con parejas ocasionales, mientras que el 6I,4\% de los jóvenes con DV reportó haber tenido al menos una relación con este tipo de pareja $\left(\mathrm{X}^{2}=\mathrm{II} .000\right.$, $\mathrm{gl}=5, \mathrm{p}=.05)$. No se encontraron diferencias por sexo en los comportamientos posteriores al primer coito sexual.

\section{- Abuso sexual}

Los resultados correspondientes a los indicadores de abuso sexual se presentan en la Tabla 4, en la cual se observa que una proporción importante de los participantes tuvieron su primera relación coital forzada y, aunque en menor proporción, también tuvieron al menos una relación forzada después de su primer coito. A un número importante de los participantes los han obligado a tocar sus genitales y los de otra persona, y los han obligado a quitarse la ropa para verlos desnudos. Al realizar un análisis cruzado entre primer coito forzado y tipo de pareja del primer coito, se encontró que de los I 8 jóvenes que indicaron que su primer coito fue forzado, en I I casos la persona con la que lo tuvieron fue el novio(a), en 3 casos fue un amigo(a), en 2 casos un familiar y en otros 2 casos una persona desconocida. El análisis de comparación por sexo mostró diferencias significativas en relaciones coitales forzadas posteriores al primer coito $\left(\mathrm{X}^{2}=3.384, \mathrm{gl}=\mathrm{I}, \mathrm{p}=.05\right)$, en obligarlos a tocar sus genitales $\left(\mathrm{X}^{2}=5.409, \mathrm{gl}=\mathrm{I}, \mathrm{p}=.02\right)$ y marginalmente significativa en obligarlos a quitarse la ropa $\left(\mathrm{X}^{2}=3 . \mathrm{I} 84, \mathrm{gl}=\mathrm{I}, \mathrm{p}=.06\right)$; en todos los casos las frecuencias fueron mayores en las mujeres que en los varones (Tabla 4). En ningún caso se encontraron diferencias significativas en estas prácticas por tipo de discapacidad de los participantes.

Tabla 4. Prácticas de abuso sexual en función del sexo de los participantes

\begin{tabular}{|l|c|c|c|c|}
\hline & $\begin{array}{c}\text { Toda la } \\
\text { muestra } \\
\text { (128) }\end{array}$ & $\begin{array}{c}\text { Hombres } \\
\mathbf{( 6 6 )}\end{array}$ & Mujeres \\
$\mathbf{( 5 8 )}$
\end{tabular}

Fuente: elaboración propia.

Por otra parte, se encontró que el 27,5\% (n=I9) de los jóvenes sexualmente activos reportó 
haber tenido un embarazo, el I4,I \% (n=9) un aborto y el $5,6 \%(\mathrm{n}=7)$ alguna ITS, aunque el 75,6\% señaló que nunca se había realizado una prueba para identificar la presencia de alguna ITS. Para conocer la asociación de estos problemas con el tipo de discapacidad y el sexo de los participantes se realizaron pruebas de Chi Cuadrada, y en ningún caso se encontraron diferencias significativas.

- Factores familiares asociados con comportamiento sexual, abuso sexual y reporte de VIH, ITS, embarazo y aborto

En toda la muestra se llevó a cabo un análisis de frecuencias a través de la prueba Chi Cuadrada para conocer la asociación de las variables sociodemográficas correspondientes a los padres, con las variables que refieren experiencia coital de los participantes, características de su primer coito, comportamiento sexual posterior al primer coito, abuso sexual y el reporte de VIH, ITS, embarazo y aborto. Los resultados mostraron que el uso de algún método anticonceptivo (MA) en el primer coito se asoció con la variable "vive tu papá", $\left(\mathrm{X}^{2}=3.562\right.$, $\mathrm{gl}=\mathrm{I}, \mathrm{p}=.05)$ y con el tipo de discapacidad del padre $\left(\mathrm{X}^{2}=8.355, \mathrm{gl}=2, \mathrm{p}=. \mathrm{OI}\right) \mathrm{y} \mathrm{de}$ la madre $\left(\mathrm{X}^{2}=8.802, \mathrm{gl}=2, \mathrm{p}=.03\right)$. Las frecuencias fueron más altas en quienes sí tienen padre $(63,8 \%)$ y en quienes el padre $(7 \mathrm{I}, \mathrm{I} \%)$ o la madre $(65 \%)$ no tiene discapacidad, comparados con los jóvenes que no tienen padre $(36,2 \%)$ y en quienes el padre $(55,6 \%)$ o la madre $(40 \%)$ tiene alguna discapacidad.

El tipo de MA utilizado en el primer coito se asoció con el tipo de discapacidad del padre $\left(\mathrm{X}^{2}=24 . \mathrm{I} 8 \mathrm{I}, \mathrm{gl}=\mathrm{IO}, \mathrm{p}=.007\right)$. La frecuencia del preservativo como MA utilizado fue más alta en los jóvenes cuyos padres no tienen discapacidad (59,I \%), que en aquellos cuyos padres tienen discapacidad visual $(55,6 \%)$. Respecto a los indicadores de abuso sexual se encontró que la variable "vive tu papá” se asoció significativamente con "alguien ha tocado tus genitales sin que tú quieras" $\left(\mathrm{X}^{2}=3.860\right.$, $\mathrm{gl}=\mathrm{I}, \mathrm{p}=.04)$ y con "alguien te ha obligado a tocar sus genitales” $\left(\mathrm{X}^{2}=5.38 \mathrm{I}, \mathrm{gl}=\mathrm{I}, \mathrm{p}=.02\right)$, con frecuencias más altas observadas en quienes no tienen padre (30\% y $20 \%$, respectivamente), comparados con quienes sí lo tienen ( I 2,4 \% y $4,7 \%$, respectivamente). En el mismo sentido, se encontraron frecuencias significativamente más altas $\left(\mathrm{X}^{2}=7.296, \mathrm{gl}=\mathrm{I}, \mathrm{p}=.007\right)$ de embarazo en jóvenes que no tienen padre $(52,9 \%)$, comparados con quienes sí lo tienen (I9,2\%). Finalmente, el nivel de estudios de la madre $\left(\mathrm{X}^{2}\right.$ $=\mathrm{I} 8.292, \mathrm{gl}=5, \mathrm{p}=.003)$ y la situación laboral del padre $\left(\mathrm{X}^{2}=8.690, \mathrm{gl}=\mathrm{I}, \mathrm{p}=.003\right) \mathrm{se}$ asociaron con el reporte de ITS, con frecuencias más altas en los jóvenes cuyas madres tienen estudios de preparatoria $(23,5 \%)$ que en madres con estudios universitarios ( $5,4 \%)$.

\section{Discusión}

Los resultados de este estudio demuestran que los jóvenes con discapacidad auditiva o visual mantienen una vida sexual activa que está caracterizada por un perfil de comportamiento sexual que los pone riesgo de infección por VIH y otras ITS, embarazo no deseado y prácticas de abuso sexual. Dicho perfil de riesgo se distingue por el inicio temprano de las relaciones coitales, la falta de planificación de su primer coito, no usar el preservativo en sus relaciones coitales y haber sufrido algún tipo de abuso sexual. Específicamente se encontró que más de la mitad de los jóvenes de la muestra había iniciado su vida sexual, con una proporción significativamente mayor en los jóvenes con discapacidad visual (DV) que en los jóvenes con discapacidad auditiva (DA). La proporción se invierte en lo relativo a la planificación del primer coito y el uso del preservativo en ese encuentro, con proporciones más altas en jóvenes con DA que no lo planificaron. Los jóvenes con DV se expusieron más a los factores de riesgo asociados con la ausencia de planificación y de uso del preservativo. El rango de la edad de la primera relación coital más frecuente en toda la muestra fue el que abarca de los i 8 a los 20 años, aunque cabe destacar que más de la mitad de los participantes inició su vida sexual 
siendo menor de edad. Dado que en este rubro no hubo diferencias en relación con el tipo de discapacidad, puede decirse que en ambos casos se pudo ubicar otro factor de riesgo que es la edad temprana de la primera relación coital.

Respecto a la edad del primer coito, no se encuentran diferencias en relación a lo reportado en investigaciones con poblaciones de jóvenes mexicanos sin discapacidad (Andrade et al., 2006; Díaz-Loving y Robles, 2009; Robles et al., 20II), en las que se ubica la edad del primer contacto sexual entre los I 5 y los I 7 años. De hecho, coincide con la edad de inicio reportada en diversos países, como lo documentan Castro et al. (2010), quienes aclaran que los adolescentes que se inician tempranamente en su actividad sexual con penetración se encuentran más tiempo expuestos al riesgo, suelen tener un mayor número de parejas sexuales a lo largo de su vida y llevan a cabo relaciones coitales sin protección.

Si se consideran los datos reportados en México por la Encuesta Nacional de Salud (Gutiérrez et al., 2013) y por investigaciones como la de Andrade et al. (2006), realizadas entre jóvenes sin discapacidad, se puede ubicar que el porcentaje de jóvenes que han iniciado su vida sexual es de alrededor del $23 \%$, con mayor número de hombres que de mujeres. En el caso de los jóvenes del presente estudio la proporción fue mayor al $60 \%$, sin diferencias por sexo de los participantes. Las diferencias en el patrón de conducta sexual se agudizan si se considera que en jóvenes sin discapacidad se ha ubicado que alrededor del $30 \%$ no utilizó preservativo en la primera relación coital, mientras que en esta investigación la proporción de jóvenes que no lo utilizaron fue mayor al $40 \%$. En ambas poblaciones, las mujeres se protegen menos que los hombres. Además, los embarazos fueron reportados en una proporción que puede considerarse alta, cerca del $30 \%$ de los jóvenes sexualmente activos dijo haber pasado por esta situación y, aunque en menor proporción, también se detectaron casos de aborto e ITS.

Es muy probable que este perfil de riesgo que caracterizó a los jóvenes con DA y DV de este estudio se deba a las serias dificultades que tienen estos jóvenes para comunicarse con la población general y para acceder a la información que podría proveerles de educación sexual, aspectos todos ellos que pueden hacer que estos jóvenes vivan sin percibir situaciones de riesgo de ITS, VIH/SIDA y embarazos no deseados, como lo reportan diversos autores (Kochung y Were, 20II; Pontes y Freitag, 20IO).

En los participantes del presente estudio pudieron detectarse otros indicadores de riesgo en comportamientos sexuales posteriores al primer coito, ya que la frecuencia del uso del preservativo en toda la vida fue menor a la frecuencia de relaciones coitales durante el mismo periodo. Puede considerarse que los jóvenes con DV están en mayor riesgo que los que tienen DA porque en los primeros es mayor la frecuencia de quienes ya tienen experiencia coital, menor la frecuencia de quienes no planificaron su primer coito y mayor la frecuencia de relaciones coitales con parejas ocasionales sin usar el preservativo. Este patrón de riesgo de los jóvenes con DV se acompañó de ciertas características sociodemográficas de sus padres, tales como la edad (que rebasa los 50 años) y un menor nivel educativo de sus madres. Dichas características podrían estar reflejando, de acuerdo con Cardenal y Fierro (200I), estilos de personalidad con mayor introversión, retraimiento y conformismo que podrían afectar la visión educativa de la sexualidad de los hijos. Melo y Zicavo (20I2) señalan que existen diversos factores que pueden considerarse obstaculizadores para el desarrollo de competencias parentales para la educación sexual y la prevención del abuso sexual hacia los hijos. Los obstaculizadores pueden ser intrínsecos y extrínsecos y, entre ellos, los autores ubican las diferencias transgeneracionales, que se refiere a la discordancia que encuentran los padres entre la forma de ver la sexualidad en generaciones anteriores y la forma en que se visualiza actualmente (tema tabú versus tema abierto, ocultamiento anterior del desnudo versus naturalidad actual). También pueden presentarse limitaciones derivadas de la propia educación sexual en la infancia, la falta de instancias de apoyo formal o la existencia de fuentes 
de aprendizaje que pueden entregar mensajes erróneos sobre sexualidad (como la televisión), pero las más recurrentes son la falta de iniciativa para prepararse, así como mostrar una actitud desprevenida y de vergüenza ante temas de sexualidad. Melo y Zicavo concluyen que los padres desarrollarán mejores competencias para la educación sexual de sus hijos en la medida en que rompan con tales obstaculizadores y asuman prácticas dirigidas a la prevención del abuso y otros riesgos de salud sexual de sus hijos.

Otros factores de influencia sobre los estilos parentales son el nivel educativo de ambos padres. Por ejemplo, Casullo y Liporace (2008) indagaron el grado en que padres y madres muestran control y castigo versus aceptación y perdón, ante comportamientos indeseados del hijo, así como el grado de consistencia en sus reacciones. Encontraron que padres y madres con educación primaria incompleta o completa mostraron mayor inconsistencia en sus estilos que los padres con niveles educativos más altos. Es muy posible que el nivel educativo, al permitir un mejor nivel de análisis y raciocinio, sea un factor que les permita a los individuos ser más consistentes en sus conductas educativas parentales, afectando positivamente la educación sexual de sus hijos.

Cabe señalar que, independientemente del sexo y tipo de discapacidad de los participantes, los factores familiares que se asociaron con conductas de riesgo fueron la ausencia del padre (no vive), su situación laboral, la presencia de discapacidad en ambos progenitores y el nivel de estudios de la madre. Estos hallazgos ponen de manifiesto la importancia que tienen los padres en el diseño de programas de intervención encaminados a prevenir problemas que afectan a la salud sexual de jóvenes con discapacidad auditiva o visual.

Un hallazgo importante de la presente investigación hace referencia al abuso sexual sufrido por los jóvenes, el cual fue medido con tres indicadores que reflejan, de acuerdo con Rodríguez et al. (20I2), tocamientos en genitales y relaciones coitales sin el consentimiento de los jóvenes. Si bien es cierto que en toda la muestra de este estudio las frecuencias de estos indicadores fueron bajas, se requiere documentar este hallazgo dada la gravedad implicada en las víctimas que sufren el abuso (González et al., 2012).

Un dato que vale la pena destacar es que i I de los I 8 jóvenes que reportaron tener su primer coito a la fuerza, dijeron que su pareja había sido su novio o novia, en tres casos fue un amigo o amiga, en dos casos un familiar y en otros dos casos una persona desconocida. Esto sugiere que los victimarios que perpetran el abuso en jóvenes con discapacidad auditiva o visual son personas que en su mayoría están cerca del ambiente en el que se relaciona la víctima y por lo mismo, de acuerdo con Finkelhor (2008), podría haber confianza y cooperación de los jóvenes hacia ellos, haciendo más probable el abuso sexual.

Finalmente, debe destacarse que las únicas diferencias debidas al sexo de los participantes se encontraron en el uso de algún método anticonceptivo en el primer coito, siendo las mujeres las que menos se protegieron, así como en relaciones coitales forzadas posteriores al primer coito y en obligarlos a tocar sus genitales y a quitarse la ropa, con frecuencias mayores en las mujeres que en los hombres. Tales diferencias, que ponen a las mujeres de este estudio en una situación de mayor vulnerabilidad al abuso sexual, podrían deberse, de acuerdo con Nosek et al. (200I), a las situaciones de sobreprotección familiar y de negación de su sexualidad que en lo general enfrenta la mujer en diferentes sociedades, así como a la negación de sus derechos humanos.

En síntesis, los resultados del presente estudio confirman lo señalado por diversos autores (Berman et al., 20I I; Guevara et al., 2016b; Robles et al., 20I4; Sargowawa et al., 2009), respecto a que los jóvenes que presentan discapacidad auditiva o visual tienen un perfil de riesgo y se encuentran en situaciones de vulnerabilidad hacia el abuso sexual repetido, hacia el ejercicio de la sexualidad sin protección y hacia ITS, VIH/SIDA y embarazo. También parece confirmarse que dicho patrón de riesgo es mayor al que puede presentarse en poblaciones 
sin discapacidad y que las mujeres son las más afectadas (Nosek et al., 200I; Smith, 2008).

La principal aportación del presente estudio es haber avanzado en la caracterización de las prácticas sexuales de jóvenes mexicanos con discapacidad visual y auditiva, así como generar datos que permiten ubicar la relación que guardan diversos factores familiares y sociodemográficos -incluyendo tipo de discapacidad-, con las prácticas de riesgo y con el abuso sexual hacia esta población.

Lo anterior se hizo posible porque se contó con instrumentos que fueron diseñados específicamente para ser aplicados con poblaciones de jóvenes con discapacidad auditiva y visual, adaptados a sus características comunicativas y encaminados a detectar factores de riesgo para su salud sexual. Contar con este tipo de instrumentos permitirá realizar estudios con poblaciones más amplias y tomar acciones preventivas y remediales que les permitan ejercer una vida sexual saludable.

Entre las limitaciones que se tuvieron para la realización del presente estudio, cabe señalar que el equipo de investigación recorrió diversas instituciones en la Ciudad de México y repetidamente se encontró con la negativa de las autoridades para dar acceso a la población de jóvenes con discapacidad. También se dio el caso de que los directivos permitieran la entrada pero los padres se negaran a que sus hijos participaran. Estos obstáculos tuvieron impacto en el tamaño de la muestra de participantes, limitando la validez externa de los resultados.

El instrumento que se utilizó para medir el abuso sexual se limitó a evaluar aspectos que refieren tocamientos y sexo con penetración sin el consentimiento del participante, pero no incluyó información sobre el victimario y su relación con la víctima, lo cual limita la comprensión del abuso sexual perpetrado en jóvenes con discapacidad auditiva o visual.

Otra limitación del estudio fue que no abordó preguntas relacionadas con las prácticas parentales. Es deseable que, en futuras investigaciones, se indague sobre estos aspectos. Se ha documentado ampliamente que existe una clara relación entre los diferentes estilos educativos parentales y diversas características y variables de los hijos, como nivel de competencia académica, estatus sociométrico, configuración de un sistema de valores, autoestima y competencia psicosocial (Torío et al., 2008). Asimismo, existe una extensa literatura que relaciona problemas de comportamiento infantil con disciplina severa, relacionada con un estilo parental autoritario, con atribuciones negativas parentales, así como con permisividad excesiva (Samaniego, 20I0). Sin embargo, poco se sabe de estas situaciones familiares en poblaciones con discapacidad. Es probable que muchos hogares en los que viven estos jóvenes muestren características como las mencionadas por Finkelhor (2008), que son comunes en las familias de los niños que son víctimas de abuso sexual.

Por último, cabe mencionar la necesidad de que, en estudios futuros, se exploren otros factores de riesgo como los conocimientos que los jóvenes con discapacidad visual o auditiva tienen acerca de diversos aspectos de su salud sexual, dadas las limitaciones comunicativas que caracterizan a esta población. 
Andrade, P. P. et al. (2006): "Factores familiares asociados a la conducta sexual en adolescentes". Revista Colombiana de Psicología, I 5: 9I- IOI.

Berman, R. et al. (20I I): "Desarrollo inclusivo: la experiencia de VIH-SIDA y discapacidad en Centroamérica". En Breve, I67: I-5.

Campero, L. et al. (2013): "Salud sexual y reproductiva de los adolescentes en México: Evidencias y propuestas”. Gaceta Médica de México, I49: 299-307.

Cantón, D. y Cortés, M. (2015): “Consecuencias del abuso sexual infantil: una revisión de las variables intervinientes". Anales de Psicología, 3 I (2): 552-56I.

Cardenal, V. y Fierro, A. (200I): "Sexo y edad en estilos de personalidad, bienestar social y adaptación social". Psicothema, I3 (I): I I 8I26.

Castro, A. et al. (2010): "Variables psicosociales que median en el debut sexual de adolescentes en España”. Revista Latinoamericana de Psicología, 43 (I): 83-94.

Casullo, M. M. y Liporace, M. F. (2008): "Percepción sobre estilos e inconsistencia parentales en adolescentes argentinos”. Estudos de Psicologia, 25 (I): 3-9.

CENSIDA (2015): Vigilancia Epidemiológica de casos de VIH/SIDA en México. Registro Nacional de Casos de SIDA. Actualización al cierre de 2015 (en línea). <http://www.gob.mx/cms/uploads/ attachment/file/2I 59I6/RN_Cierre_20I 5.pdf >, acceso 24 de mayo de 2017.

Díaz-Loving, R. y Robles, S. (2009): “Atracción, romance, sexo y protección en adolescentes”. Enseñanza e Investigación en Psicología, I4 (2): $215-228$.

Enríquez, D. et al. (2005): "Variables relativas al uso del condón en hombres y mujeres universitarios”. Revista Electrónica de Psicología Iztacala, 8 (2): 8I-98.
Ferreira, W. B. (2008): "Vulnerabilidade á violence sexual no contexto da escola inclusiva: reflexao sobre a invisibilidade da pessoa como deficiencia". Revista Electrónica Iberoamericana sobre Calidad, Eficiencia y Cambio en Educación, 6 (2): I 20-I36.

Finkelhor, D. (2009): "The prevention of childhood sexual abuse". The Future of Children, I9 (2): I69-I94.

Finkelhor, D. (2008): Abuso sexual al menor. México: Pax.

García, P. y Nastri, M. (20 I I): "Sexualidad en adolescentes con discapacidades motoras". Archivos Argentinos de Pediatría, Io9 (5): 447452 .

González, E. et al. (2OI2): "La conducta sexual infantil como indicador de abusos sexuales: los criterios y sesgos de los profesionales". Psicothema, 24 (3): 402-409.

Guevara, Y. et al. (2016a): Educación sexual en personas con discapacidad. Fundamentos para talleres de intervención. México: Facultad de Estudios Superiores Iztacala. Universidad Nacional Autónoma de México.

Guevara, Y. et al. (2016b): "Comunicación con padres, comportamiento sexual, e indicadores de abuso sexual en jóvenes mexicanos con discapacidad visual". Psychologia: avances de la disciplina, Iо (I): 79-90.

Gutiérrez, J. P. et al. (20I3): Encuesta Nacional de Salud y Nutrición 20I2. Resultados nacionales. Cuernavaca, México: Instituto Nacional de Salud Pública (en línea). <http://ensanut.insp.mx/informes/ ENSANUT20 I 2 ResultadosNacionales2Ed. pdf>, acceso 24 de mayo de 2017.

Instituto Nacional de Estadística, Geografía e Informática, INEGI (20I 5): México. Encuesta intercensal (en línea). <http://www.inegi.org. $\mathrm{mx} / \mathrm{est} / \mathrm{contenidos/proyectos/encuestas/hogares/}$ especiales/eizo I 5/default.aspx>, acceso 24 de mayo de 2017. 
Kochung, E. y Were, M. (2OII): "Self perception on risk factors associated with HIV/AIDS infection among the blind youth in tertiary colleges". Educational Research, 2 (I): 943-947.

López Sánchez, F. et al. (20I I): "Diferencias sexuales en la sexualidad adolescente: afectos y conductas”. Anales de Psicología, 27 (3): 79 I799.

Melo, M. y Zicavo, N. (20I2): "Competencias parentales en educación sexual". Ciencias Psicológicas, VI (2): I23-I33.

Méndez, I. et al. (200I): El protocolo de investigación. Lineamientos para su elaboración $y$ análisis. México: Trillas.

Moinester, M. et al. (2008): "The nature of risk. HIV/AIDS and the deaf community in the United States”. Disability Studies Quarterly, 28 (4).

Moreno, D. et al. (2008): "Características del debut sexual de los adolescentes y determinantes del uso consistente del condón desde el análisis contingencial”. Psicología y Salud, I 8 (2): 207225 .

Muñoz-Rivas, M. et al. (2009): "Prevalence and predictors of sexual aggression in dating relationships of adolescents and young adults". Psicothema, 2I (2): 234-240.

Nosek, M. et al. (200I): "Vulnerabilities for abuse among women with disabilities". Sexuality and Disability, I9 (3): I77-I89.

Ocampos, C. (2005): “Abuso sexual en la infancia y adolescencia”. Pediatría, 32 (2): 25-29.

Organización Mundial de la Salud (2016a). Adolescentes: riesgos para la salud y soluciones (en línea). <http://www.who.int/mediacentre/ factsheets/fs345/es/>, acceso 24 de mayo de 2017.

Organización Mundial de la Salud (2016b). Maltrato infantil (en línea). <http://www.who. int/mediacentre/factsheets/fs I $50 /$ s/ $/>$, acceso 24 de mayo de 2017.

Parchomiuk, M. (20I4): "Social context of disabled parenting". Sexuality and Disability, $32(2): 23$ I-242.
Pontes, C. y Freitag, L. (2010): “The experience of sexuality by visually impaired adolescents". Revista da Escola de Enfermagem da USP, 44 (3): $577-582$.

Robles, S. et al. (2015): Factores de riesgo para la salud sexual de jóvenes con discapacidad auditiva y visual: un estudio exploratorio (informe técnico final). DGAPA-UNAM, proyecto $\mathrm{IN}_{3} \mathrm{O} 8 \mathrm{I}_{3} 3$.

Robles, S. et al. (20I4): "Conductas sexuales de riesgo en jóvenes con discapacidad auditiva", en Rivera, S. et al. (eds.): La psicología social en México. Volumen XV. México: Asociación Mexicana de Psicología Social.

Robles, S. et al. (2013): "Comunicación con padres y conducta sexual en jóvenes mexicanos con discapacidad auditiva”. Revista Psicología y Salud, 23 (2): 227-239.

Robles, S. y Díaz-Loving, R. (20 I I): Validación de la Encuesta Estudiantil sobre Salud Sexual (EESS). México: Universidad Nacional Autónoma de México.

Robles, S. et al. (20I I): "Conocimientos sobre VIH/SIDA, comunicación sexual y negociación del uso del condón en adolescentes sexualmente activos”. Revista Electrónica de Psicología Iztacala, I4 (4): 3 I7-340.

Robles, S. et al. (2006): "Entrenamiento conductual en habilidades de comunicación sexual en la pareja y uso correcto del condón”. Anales de Psicología, 22 (I): 60-7I.

Rodríguez, Y. et al. (20I2): "Consecuencias psicológicas del abuso sexual infantil”. Eureca, 9 (I): 58-68.

Sakellariou, D. (2006): "If not the disability, then what? Barriers to reclaiming sexuality following spinal cord injury". Sexuality and Disability, 24 (2): IOI-III.

Samaniego, V. (2010): Escala de tolerancia parental hacia los comportamientos infantiles, elaboración y validación. Revista Latinoamericana de Psicología, 42 (2): 203-2I4.

Santín, L. (20I2): Abuso sexual y discapacidad intelectual (tesis inédita de Maestría en Políticas Sociales de la Infancia y Adolescencia). 
Universidad Politécnica Salesiana, Quito, Ecuador.

Sargowawa, A. et al. (2009): "Sexual practices of deaf and hearing secondary school students in Ibadan, Nigeria". Annals of Ibadan Postgraduates Medicine, 7 (I): 26-30.

Save the Children (2003): Sexual abuse of children with disabilities (en línea). <http:// resourcecentre.savethechildren.se/sites/default/ files/documents/docs-_22867-vI-sexual_abuse_ of_disabled_children_-_summaries_from_three_ reportsI_o.pdf>, acceso 24 de mayo de 2017.

Secretaría de Salud de la Ciudad de México (20I7): Interrupción legal del embarazo
(ILE). Estadísticas abril 2007 - I7 de mayo 2017 (en línea). <http://ile.salud.cdmx.gob. $\mathrm{mx} / \mathrm{wp}$-content/uploads/Interrupcion-Legaldel-Embarazo-Estadisticas-2007-20 I 7-I 7demayo20I7.pdf>, acceso 25 de mayo de 2017.

Smith, D. (2008): “Disability, gender and intimate partner violence: Relationships from the behavioral risk factor surveillance system". Sexuality and Disability, 26 (I): I 5-28.

Torío, S. et al. (2008): "Estilos de educación familiar”. Psicothema, 20 (I): 62-70.

Verdugo, M. et al. (2002): "El abuso sexual en personas con discapacidad intelectual". Psycothema, I4 (suplemento): I24-I 29. 